\title{
ITF

\section{Social media, learning, networking and promotion opportunities for tennis coaches}

\author{
Mauricio Córdova (ECU) \\ ITF Coaching and Sport Science Review 2016; 68 (24): 6 - 8
}

\begin{abstract}
This article examines three trends that will shape the future of social media. From these innovations, five practical tools addressed to coaches with little experience in the use of social media are proposed. These resources are focused on the promotion of clubs or academies and professional career development through networking and self-learning. The latest innovations and trends in social media will be explored and from this, five recommendations for utilisation by tennis coaches will be proposed.
\end{abstract}

\author{
Key words: social media, \\ promotion, new technologies, \\ career development \\ Received: 05 October 2015 \\ Accepted: 10 January 2016 \\ Corresponding author: \\ Mauricio Córdova \\ Email: mauc875@gmail.com
}

\section{INTRODUCTION}

Nowadays, social media has transformed from being a simple space for entertainment, to becoming a meaningful resource for companies and organisations that seek to establish closer relationships with their groups of interest. Tennis is not alien to this reality, for coaches and academies are increasingly taking advantage of social media as a means of promotion and dissemination of knowledge.

A key element to understanding the current situation on social media is the new communication paradigm. As suggested by Weinberger, Locke, Levine, and McKee, (2009) it is suggested that communication ceases to be unidirectional to be transformed in a conversation where human and direct language prevails. A second characteristic is the fast level of innovation in the operative systems as well as in the mobile devices that have permitted a higher number of users to be interconnected.

\section{CURRENT TRENDS IN THE USE OF SOCIAL MEDIA \\ The revolution of mobile devices}

The abundance of smart phones and tablets is current markers is increasingly high. Kemp (2015), showed that internet traffic produced by fixed devices (laptops and desktops) has decreased $13 \%$ and hence, mobile devices now account for $38 \%$ of overall web traffic. In this sense, Kemp (2015) and Bullas (2015) emphasised that around $29 \%$ of the world's population has at least one active social media account. It is estimated that currently $79 \%$ of these users, roughly equating to 1.65 billion people, are accessing these applications through mobile devices. Moreover, to note the level of dependency on such mediums, on average $17 \%$ of social media users check their devices for three or more hours per day (Cooper, 2013). The boom and consolidation of instant messaging through applications such as WhatsApp, Telegram or Snapchat also suggests the rise of a new era in the use of social media.

\section{A wide variety of resources}

The level of diversification on social media networking sites keeps growing. Although Facebook remains as the largest platform worldwide with almost 1400 million users (Bullas, 2015), other tools do also present an immense potential. Linkedln, for example, makes it possible for coaches to interact with National/International Associations and sporting bodies, as well as other coaches and professionals from around the world. This business-oriented social networking service is primarily beneficial in supporting professionals with further career opportunities, offering professional services and facilitating discussions with experts in various fields. It is estimated that each second, two new users log into this social media site (Cooper, 2013).

YouTube closely follows Facebook in terms of the number of active users in the world. To gain an insight into its impact, (Cooper, 2013) highlighted that in the United States this platform has higher audience levels than any TV cable channel amongst young people

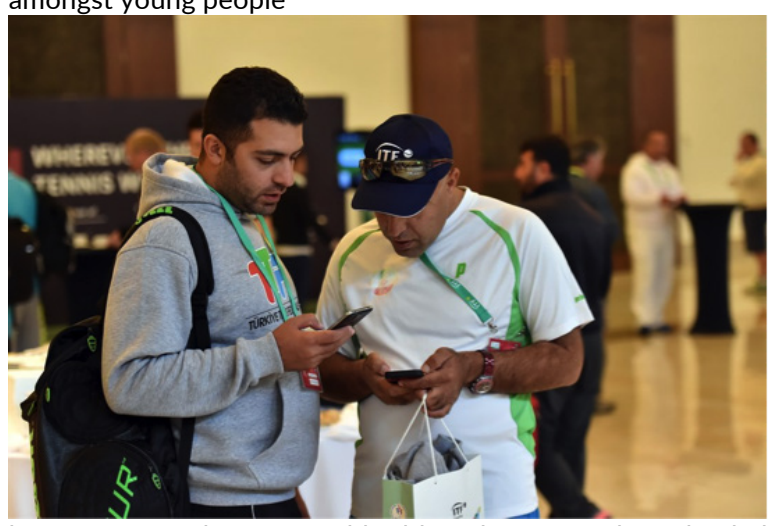

between 18 and 34 years old. Although most of the uploaded content on this YouTube is entertainment oriented, its potential as an educational tool for sharing knowledge has often been disregarded. From a tennis coaching perspective, this platform may seem quite attractive given the comprehensive amount of free available resources such as tutorials, speeches, and point sequences among others.

Another social networking site that has gained mass amounts of popularity over the last few years is Instagram, an online mobile photo/video-sharing platform where users can upload and share high quality images and other visual resources on various social network websites. Lunden (2014) points out that this site grew in 2014 more than any other social media outlet with an almost $23 \%$ growth. YouTube and Instagram are quite relevant as the current contents trends suggest that most of the conversations on social media no longer solely depend on traditional or written communication forms. Ayres (2014) estimated that at least $87 \%$ of shared contents on Facebook was the result of a good image. Moreover, Codefuel (2015) 
forecasted that in 2017 , nearly $74 \%$ of the overall traffic on the internet will be generated from videos.

\section{Group interactions}

According to the new communication paradigm, it is becoming increasingly more frequent that dynamics of interaction are becoming more granular, meaning individuals are now seeking more compact and homogenous groups where common interests and values can be met. For instance, the group "Tennis Industry Network" on Linkedln gathers more than 9,000 experts on tennis worldwide, most of them active professionals in the field. Stets y Burke (2000) argued that a key factor in this new networking approach is that individuals in a group would identify themselves more openly in spaces where they could perceive a positive self- image. Therefore, the closer the relationship between a group and a person is, the greater the disposition to participate and interact.

\section{FIVE BASIC TOOLS FOR COACHES BEGINNING IN SOCIAL} MEDIA

From the trends that have just been outlined, five basic tools are presented for coaches that can be used in order to take advantage of these resources as a means of promoting local activities, as well as to continually develop a professional career in tennis.

Start with a Facebook group for your club or academy

Currently, Facebook offers three types of accounts: personal pages, public pages and groups. For clubs and academies that are being promoted for the first time, it is highly advisable to start by means of a group page approach. The main advantage of this feature is that it permits more contributions by group members as it is possible for them to directly post photos, videos and other files.

\section{Recommendations:}

- When opening a group, clearly set the privacy levels by keeping control of the type of group; whether it can be public, open or secret, and any restrictions you would like to put on the posts of the members.

- It is paramount to state a detailed description of the group and to choose an appealing page photo that at a first glance will clearly portray who the group are and their main purpose/s.

- The posts should not be repetitive and, if possible, they should be visually attractive. Be sure to take advantage of the events tool when a course, exhibition, tournament, ranking event or any other activity takes place on your club.

- Keep in mind that the group updates when a new entry or comment has been posted and goes directly to the notifications section in members' personal pages. Thus, excessive and irrelevant posts may decrease the interest in the group.

- An active group with a good level of content can be time consuming. If you are willing to accept this challenge as group administrator, you should be ready to respond as soon as possible to comments and questions from group members.

Improve your designs with these free online resources

Visual content is a fundamental component in social media. To create posters, banners and infographics, we advise you to use the following resources which might be helpful when, for instance, organizing a local tournament or any other activity at your club:

Visual content is a fundamental component in social media. To create posters, banners and infographics, the following resources may be helpful when, for instance, organising a local tournament or any other activity at your club:

Images: https://www.canva.com

Infographics:
- Try to be consistent in the colour patterns used towards defining a corporate line to your club and hence to your page. - Bear in mind that currently, most of the content on social media are being spread through mobile devices. With these design tools it is possible to adjust or adapt the sizes to these types of formats.

- As the saying goes, an image is worth one thousand words. One of the keys for posting good content is to have a wide and rich photo base. Be sure that there is always photographic evidence of the important moments at your club. The quality of pictures is also decisive so do not feel the need to spare any expense when investing in a good camera.

- Post weekly rankings, an interesting statistic or advice from a successful coach or player.

Start producing your own videos

Creating and editing videos is much simpler than it actually looks. Although YouTube is an endless source of information and

entertainment, not many users use this platform to generate their own content. A recommended tool for creating these types of contents is the YouTube editor itself (https://www.youtube.com/ editor).

Recommendations:

- Be sure to always name your videos with an appealing name, labelling them with key words that would permit other users with similar interests to find your clip. Do not neglect the huge potential of this social networking site. Just imagine that if YouTube was a nation, it would probably be the third largest in the world behind China and India!

- It is possible to include free copyright music in your videos with this editor. Do not forget to pick a good soundtrack to enhance your clip.

- It is highly advisable that the video should be shorter than one minute.

- Combine your clip with images and text. Besides if you wish to widen your audience, YouTube editor permits you to add subtitles in other languages automatically.

- In this link you may find a quick tutorial on how to edit your first video:

https://www.youtube.com/watch?v=ZYK2p7MZQqw

Strengthen your career path on LinkedIn

This suggestion is rather focused on improving your professional image in the tennis industry as a coach. Through Linkedln, you will be able to expand your network with other experts in the field as well as taking advantage of calls, working opportunities and new learning resources that may contribute to your professional development.

- When creating your account, only include relevant information that supports your current professional exploits.

- A clear and professional photo is fundamental as well. As in a real CV, this will be the first impression you are going to project. - Be sure to follow Linkedln pages of tournaments, clubs, academies and National Federations where courses and working opportunities are often published.

- As this is a professional network, politeness and your language style is highly important. Carefully select the information you are sharing or posting and avoid making spelling mistakes.

Take part actively in tennis groups

Jenkins, Ford and Green (2013) stress that belonging to a group or network means to have a mutual power, foundation of the so- called "participatory culture". Thus, one of the main principles when venturing into the world of social media is that the best approach to interact is by sharing. 
Recommendations:

- Contributing actively in other groups is an excellent strategy to promote yourself as a coach and at the same time to spread the services of your club or academy.

- It may be encouraged for you to propose topics of discussion for group members which should be relevant. Nonetheless, you should also concede importance to the discussion opened by the other participants.

- It can also be suggested that you join one of the following groups where you can meet tennis experts and find innovative resources for your professional growth.

Linkedln: (Each group goes with hyperlink):

- Professional tennis coaches

https://www.linkedin.com/grp/home?gid=8132140\&sort=PO PULAR\&trkInfo=clickedVertical\%3Agroup\%2CclickedEntityld \%3A8132140\%2Cidx\%3A3-3-

8\%2Ctarld\%3A1443012474920\%2Ctas\%3Acoaches+tenn\&tr k=tyah

- ITF Coaching

https://www.linkedin.com/groups?gid=8112630\&trk=vsrp groups_res_name\&trklnfo=VSRPsearchld\%3A530503931443 012607106\%2CVSRPtargetld\%3A8112630\%2CVSRPcmpt\% 3Aprimary

- Tennis industry Network

https://www.linkedin.com/groups?gid=2350552\&trk=vsrp_

groups_res_name\&trklnfo=VSRPsearchld\%3A53050

3931443012819908\%2CVSRPtargetld\%3A2350552\%

2CVSRPcmpt\%3Aprimary

- Jobs and Careers in tennis

https://www.linkedin.com/groups?gid=2692589\&trk=vsrp_ groups_res_name\&trklnfo=VSRPsearchld\%3A530503931443 012819908\%2CVSRPtargetld\%3A2692589\%2CVSRPcmpt\%

3Aprimary

-Strength and conditioning for tennis https://www.linkedin.com/grp/home?gid=4440467

- The business of tennis

https://www.linkedin.com/groups?gid=3672620\&trk=vsrp groups_res_name\&trkInfo=VSRPsearchld\%3A530503931443 012819908\%2CVSRPtargetld\%3A3672620\%2CVSRPcmpt\% 3Aprimary

Facebook: (Each group goes with hyperlink)

- Real Tennis Coaches

https://www.facebook.com/groups/643197515728943/?ref $=$ ts \&fref $=$ ts

- British Tennis Coaches Forum

https://www.facebook.com/groups/BritishTennisCoachesFor u $\mathrm{m} /$ ?ref=ts\&fref=ts

- Tennis Coaches Worldwide

https://www.facebook.com/groups/29343537440/?ref=ts\&f

ref $=$ ts

- Competitive Tennis Coaching

https://www.facebook.com/groups/justtennisplayers/?ref=br - rs

\section{CONCLUSION}

Social networking sites have become essential resources in the changing, Globalized and competitive environment we currently live in, including the tennis industry. This article has presented current trends in the use of new technologies and from this momentum, five practical, manageable and free of cost tools were recommended for coaches and directors of clubs that seek to start exploring its immense potential.

\section{REFERENCES}

Ayres, S. (2013). Social Media facts, Retrieved September 8, 2015 from http://www.postplanner.com/crazysocial-media-facts- that-are-actually-true/

Bullas, J. (2015). 33 Social Media Facts and Statistics You Should Know in 2015, Retrieved September 8, 2015 from https://www. linkedin.com/pulse/33-socialmedia-facts-statistics-you- should-know-2015-jeffbullas/

Cooper, B. (2013). Social Media statistics, Retrieved September 8, 2015 from https://blog.bufferapp.com/10surprising-socialmedia-statistics-that-will-make- you-rethink-yourstrategy

CMO COUNCIL. (2013). Internet Marketing, Retrieved September 11, 2015 from http://www.cmocouncil.orgfacts-stats-categories. php?category=internet- marketing

Kemp, S. (2015). Digital, Social \& Mobile Worldwide in 2015, Retrieved September 13, 2015 from http://wearesocial.net/ blog/2015/01/digitalsocial-mobile- worldwide-2015/

Lunden, I. (2014). Instagram The fastest growing social site, Retrieved September 18, 2015 from http://techcrunch.com/2014/01/21/ instagram-isthe-fastest- growing-social-site-globally-mobiledevices-rule-over-pcs-for-social-access/

Reed, R. (2014). The WhatsApp Architecture Facebook, Retrieved September 11, 2015 from http://highscalability.com/ blog/2014/2/26/thewhatsapp-architecture- facebook-bought- for-19billion.html

Stets, J.E., \& Burke, P.J. (2000). Identity theory and social identity theory. Social Psychology Quarterly, 63(3), 224-237.https://doi.org/10.2307/2695870

RECOMMENDED ITF TENNIS ACADEMY CONTENT (CLICK BELOW)

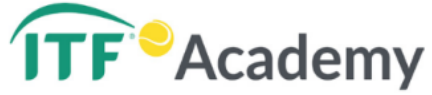

Copyright (c) Mauricio Córdova 2016

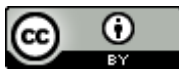

This text is under a Creative Commons BY 4.0 license

You are free to Share - copy and redistribute the material in any medium or format - and Adapt the content - remix, transform, and build upon the material for any purpose, even commercially under the following terms:

Attribution: You must give appropriate credit, provide a link to the license, and indicate if changes were made. You may do so in any reasonable manner, but not in any way that suggests the licensor endorses you or your use.

CC BY 4.0 license terms summary $\quad$ CC BY 4.0 license terms 